\title{
Lymphocyte levels following COVID-19 vaccine BNT162b2
}

Oren Miron, M.A., Nadav Davidovitch, M.D., M.P.H

Author affiliation: Department of Health Policy and Management, Ben-Gurion

University, Beer Sheva, Israel (Miron and Davidovitch). Corresponding author:

Oren Miron, MA, Department of Health Policy and Management, Ben-Gurion

University, Beer Sheva, Israel (orenmir@ post.bgu.ac.il).

Keyword: COVID-19 Research Database; COVID-19; SARS-CoV-2;

BNT162b2; mRNA vaccine; Lymphocyte

\section{Introduction}

The BNT162b2 vaccine has been shown to be effective in reducing the incidence, severity and mortality of Coronavirus Disease 2019 (COVID-19). ${ }^{1,2}$ The clinical trial report of BNT162b2 suggested that the mechanism of BNT162b2 includes lymphocytes migration from the blood to the lymph nodes, and that it relates to the clinical trial finding of decreased blood lymphocytes in the 3 days following dose- 1 of BNT162b2. ${ }^{3}$ A decrease in blood lymphocytes was also shown in the second day after dose-1 BNT162b2 in another study, and in studies of BNT162b1 and other mRNA vaccines. ${ }^{4}$ The BNT162b2 clinical trial also found that lymphocytes were normal in the 6-8 days following dose-1 and dose-2, but it did not test lymphocytes in the 3 days following dose-2. Our 
study aims to estimate the lymphocytes in the 3 days following dose- 2 using existing electronic health records, to help improve the understanding of the BNT162b2 dose-2 mechanism.

\section{Methods}

We extracted values of lymphocyte blood tests and BNT162b2 immunization from Electronic Health Record data including diagnosis, procedures, labs, vitals, medications and histories sourced from participating members of the Healthjump network, which is situated in the United States. Absolute lymphocytes were calculated as $10^{\wedge} 3 / \mathrm{mm} 3$ (thousands per cubic millimeter), and vaccines were extracted from December $10^{\text {th }} 2020$ to September $30^{\text {th }} 2021$ based on the CXV code 208, the CPT code 91300 , and the vaccine description from a database of 900 thousand vaccinations. ${ }^{5}$ We included BNT162b2 first dose administration and second dose BNT162b2 administrations that were done 21 days after a BNT162b2 first dose administration to resemble the clinical trial protocol (excluding dose-2 before or after day 21). We calculated the median lymphocyte values in each of the 14 days before and after the vaccination to determine its lowest median value, and we also compared the lymphocyte values in the 3 days before and after the administration using Wilcox rank test (significance at $\mathrm{p}<0.05$ ). 


\section{Results}

We extracted 13,329 records, with a median age of 63 years. The median lymphocyte in the 14 days before the $1^{\text {st }}$ and $2^{\text {nd }}$ dose was $1.85\left(10^{\wedge} 3 / \mathrm{mm} 3\right)$. For the first dose, the lowest median value was $1.75\left(10^{\wedge} 3 / \mathrm{mm} 3\right)$ and it was reached 3 days after administration, while for the second dose the lowest median value was $1.35\left(10^{\wedge} 3 / \mathrm{mm} 3\right)$ and it was reached 2 days after administration (figure 1).

Figure 1: Lymphocytes by day from BNT162b2 administration $(\mathrm{N}=13,329)$

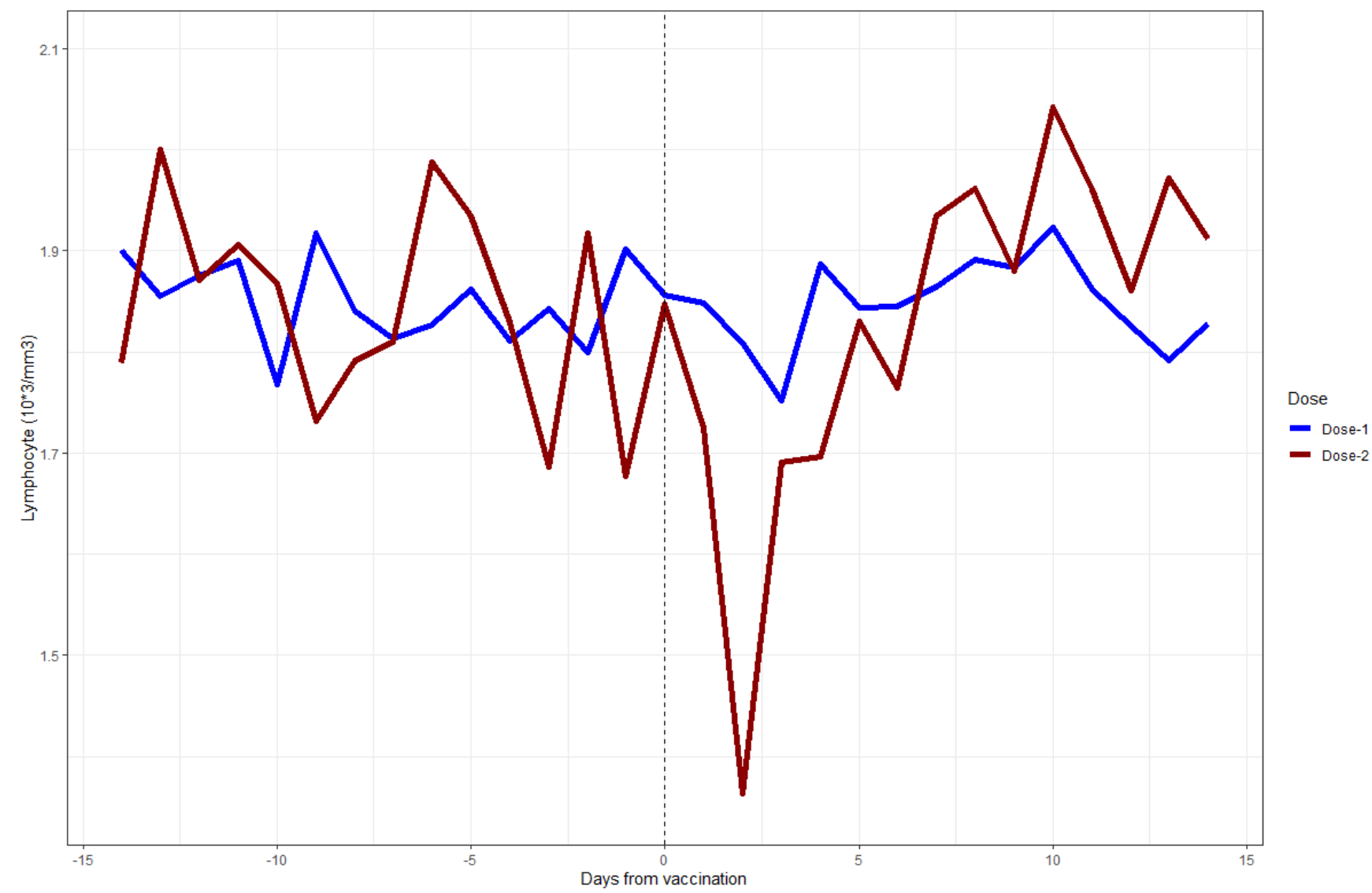

Legend: Y-axis indicates median lymphocyte blood levels $\left(10^{\wedge} 3 / \mathrm{mm} 3\right)$, while $\mathrm{X}$-axis indicates the days from administrating the BNT162b2 vaccine. Blue line indicates the first dose, and the red line indicates the second dose. 
The lymphocyte value in the 3 days after dose- 2 administration were significantly higher than those in the 3 days before the vaccination $(\mathrm{p}<0.01$; figure 2).

Figure 2: Lymphocytes in the 3 days before and after BNT162b2 administration

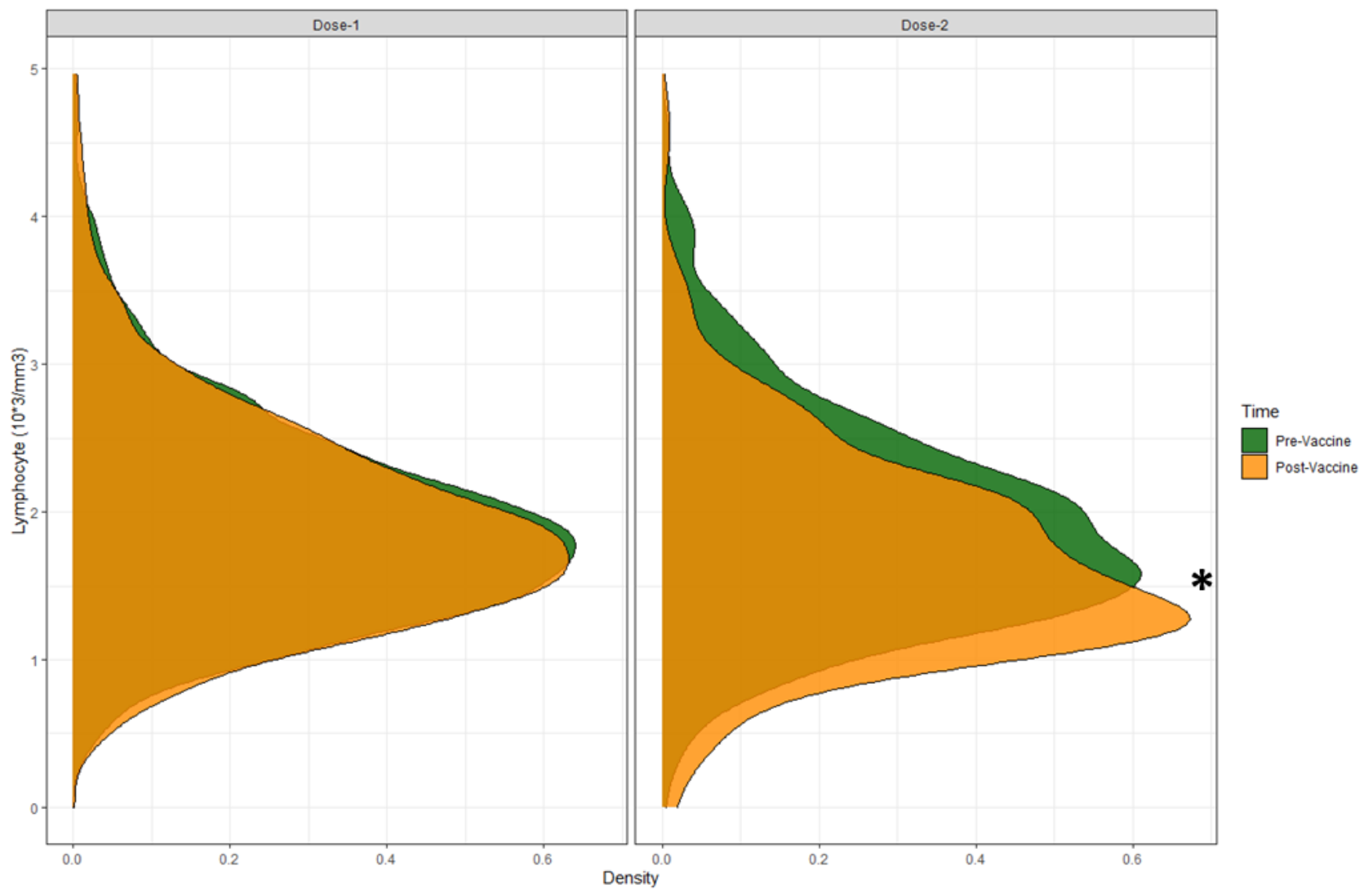

Legend: Y-axis indicates median lymphocyte blood levels (upper limit of 5 $10 * 3 / \mathrm{mm} 3$ ), while $\mathrm{X}$-axis indicates the proportion of tests that have the given value. Green indicates the 3 days before the administration of BNT162b2 and the orange indicates the 3 days following the administration. The left panel 
indicates dose-1 and the right panel indicates dose-2. Asterisks $(*)$ denote $<0.05$.

\section{Discussion}

Our analysis suggests that lymphocyte blood levels have a temporary decrease in the 3 days following the second dose of BNT162b, which resembles the decrease reported after the first dose in the clinical trial. This could relate to the high increase in antibodies that is often found following the second dose.

The main limitation of the study is that the lymphocyte tests were done for a medical reason, such as an annual exam or to diagnose a disease, unlike the clinical trial that tested each participant.

Future vaccine studies could examine blood lymphocytes in the 3 days following the second dose to verify this finding and further examine the dose- 2 mechanism and its effect. 
Acknowledgments: The data, technology, and services used in the generation of these research findings were generously supplied pro bono by the COVID-19 Research Database partners, who are acknowledged at https://covid19researchdatabase.org/.

Author Contributions: Oren Miron had full access to all of the data in the study and takes responsibility for the integrity of the data and the accuracy of the data analysis.

Concept and design: All authors.

Acquisition, analysis, or interpretation of data: All authors.

Drafting of the manuscript: Miron.

Critical revision of the manuscript for important intellectual content: Davidovitch.

Statistical analysis: Miron.

Study supervision: Davidovitch.

Conflict of Interest Disclosures: None declared.

Funding/Support: None. 
1. Polack FP, Thomas SJ, Kitchin N, et al. Safety and efficacy of the BNT162b2 mRNA Covid-19 vaccine. New England Journal of Medicine. 2020;383(27):2603-2615. doi:10.1056/NEJMc2036242

2. Dong E, Du H, Gardner L. An interactive web-based dashboard to track COVID-19 in real time. The Lancet infectious diseases. 2020;20(5):533-534.

3. Walsh EE, Frenck Jr RW, Falsey AR, et al. Safety and immunogenicity of two RNA-based Covid-19 vaccine candidates. New England Journal of Medicine. 2020;383(25):2439-2450.

4. Sahin U, Muik A, Vogler I, et al. BNT162b2 vaccine induces neutralizing antibodies and polyspecific T cells in humans. Nature. Published online 2021:1-6.

5. Breakthrough Infections Following Vaccination Against COVID-19: Descriptive Statistics From the COVID-19 Research Database. COVID-19 Research Database. Published September 2, 2021. Accessed November 12, 2021. https://covid19researchdatabase.org/breakthrough-infectionsfollowing-vaccination-against-covid-19-descriptive-statistics-from-the-covid-19-researchdatabase/ 